\title{
The balanced diet during the stall period as sheep dermatitis preventing factor
}

\author{
Bagama Bagamaev ${ }^{1}$, Eduard Gorchakov ${ }^{1}$, Natalia Fedota ${ }^{1,},{ }^{*}$, Nikolai Gvozdetsky ${ }^{1}$, Nadegda \\ Taranuha ${ }^{1}$, and Natalia Kolomysova ${ }^{1}$ \\ ${ }^{1}$ Stavropol State Agrarian University, Zootekhnicheskiy Alley, 12, Stavropol, Russia
}

\begin{abstract}
The intensity and direction of the living organism's metabolic processes determine the growth rate, development, nutrients synthesis, as well as the reproductive body function and the biosynthesis of proteins, fats and carbohydrates in the form of feedstuff and raw materials. The higher animals' productivity, the more intensive are metabolic processes. The living bodies' metabolism proceeds with a certain speed and at the same time contrariwise, but with accurate coherence and interaction due to the participation of "life regulators", enzymes, which represent the specific proteins, the activity of which is regulated by hormones, vitamins and various synergistic elements. Chemical elements included in the living organism structure are not synthesized in it but must be originated from feeds and other nutrients. Changes in essential micronutrients supply shall not be studied in isolation from the general bio-geochemical situation. Both excess and deficiency may lead to metabolic disorders involving enzyme systems. Knowledge applied by veterinary specialists about the imbalance of various chemical elements in soil, water and feeds has formed the basis for raising the issue of endemic animal diseases diagnosis and prevention. The feeds inadequacy often leads to undernutrition and the reduction of their value in the stall period, which causes the fatness reduction and, therefore, the decrease in mobility and activity, and the weakness of animals. General inspection and palpation of animals reveal ruffled coat, dry-looking and non-supple skin, and if the lack of nutrients becomes more intense, anemic mucous membranes (in more complicated forms even cyanosis and icterus) are observed.
\end{abstract}

\section{Introduction}

The provision of country residents with healthy foods and manufacturing and processing industry with up-to-date raw materials is only possible in case of constant animal husbandry monitoring and developing, with the increase in high-yield cattle.In this respect, diseases appearing from the series of causes, including metabolic disorders, constitute the key problem. The substantive reason of animals' metabolism changes is inadequate, unbalanced feeding including problems with minerals [1-6].

There are over 70 chemical elements of the periodic table present in the animal body,

\footnotetext{
* Corresponding author: nataliafedota@yandex.ru
} 
including micro- and ultranutrients, the content of which amounts to under one per cent, and in many cases even under one thousandth of body weight. However, many of them are important and necessary for biochemical processes maintenance.

At the present day the biological implication is studied and proved of many macro- and micronutrients. As well as being the structural components of living organisms, many elements also catalyze the biochemical processes in the cell, as they are present in coenzymes, enzymes, hormones, vitamins or various chemical systems activators.

Heavy yielders and their constant reproductive function determine the intense metabolic processes in cells, organs, tissues and in the whole organism in general. The supply with the stock diet of strictly required doses of nutrients critical for metabolic processes is the essential requirement for the provision of optimal physiologically founded protein anabolism in the organism, energy balance, quick growth and organism development, necessary to produce milk, meat and other products of animal origin of the required quality. Animal management, including the environment and exercises, is another substantive factor influencing metabolic processes.

Diet violation and housing conditions not meeting the physiological needs of all types of productive animals cause various disturbances of metabolic processes which lead to the decrease of resistance, clinically apparent effects and diseases of mature animals and young stock.

Systematic observations of the number of animals in the stall period revealed that the first half of winter in the absence of pasture content gradually increased the number of patients with signs of tousled coat, followed by the manifestation of itching and hair loss. Crowding of livestock and manifestations of dermatitis leads to a decrease in productivity, weakening of the animal up to the case.

The transfer of young animals from cells to groups with ewes leads to an increase in contact with clinical sick animals, this leads to a rapid spread of the disease in groups than among adults. This course of morbidity in most cases occurs first hidden and lightning-fast form (within a few days to one week). Later the lambs manifested a clinical picture similar to adult individuals.

One of the reasons of sheep's skin diseases is changes in metabolic processes resulting from insufficient diet and nutritional deficiency, including the lack of vitamins, macro- and micronutrients necessary for the adequate diet.

The soil cover of livestock farms in the most regions of Stavropol Krai contains the increased amount of calcium and iron, and lacks phosphorus, copper and selenium. The study was pursued of the chemical content of sheep's food supply. Analytical studies showed that in the autumn-winter period sheep blood reveals the excess of calcium and lack of phosphorus $[1,7,8]$.

\section{Materials and methods}

Research of the chemical composition of feedstuffs for presence of amino acid composition was conducted using the amino acid analyzer AAA-400 (INGOS s.r.o., Czech Republic).

Biochemical research of animal blood was conducted using the biochemical blood analyzer Stat Fax 3300 (AWARENESS Technology Inc., USA), and hematology analyzer Abacus Junior Vet (Diatron, Austria).

Mineral composition of feedstuffs for presence of calcium and phosphorus was conducted using vacuum wavelength-dispersive X-ray fluorescent spectrometer designed for detecting chemical elements Spectroscan Max GV (SPEKTRON Research and Manufacturing Association, LLC, Saint Petersburg, Russia).

The research was conducted using 146 fine-wool sheep and 160 lambs, of which two groups have been formed, a test group and a control group. During the first stage, we 
estimated overall clinical condition of the animals (thermometry, pulse and respiratory rate measurement). Throughout the research, we monitored liveweight gain dynamics by carrying out weighing on a weekly basis. During the second stage of the experiment, we conducted hematologic and biochemical research of animal blood of both test and control group to reveal reference data.

\section{Results}

The imbalanced feeding diet leads to the problems in animals' metabolism biochemistry and protective function depression, which often results in many diseases with productive ability reduction and even death of animals. For this reason, the examination of nutrient value of animals feed followed by the detection of its composition, both organic and mineral, poses one of topical issues.

Feed researches using current chemical analytical analysis methods revealed that the feed used in the stall period led to changes in the level of consumption by animals of minerals. Biochemical studiesof sheep detected the excess amount of calcium and the lack of phosphorus, typical for extremely dry and dry regions of Stavropol Krai. Besides, the most sufficient changes in phosphorus supply is observed in extremely dry region, which leads to its minimal flow to the animal organism. Literature sources show that the optimum ratio for sheep in calcium and phosphorus is $1.5: 2$. In lambing period,ratioCa:P $(1: 1)$ [ 1 , 9] is recommended to keep the balance of calcium and phosphorus. However, the diet used in dry and extremely dry regions does not provide such ratio.

The imbalance in the diet of calcium and phosphorus leads to sharp change in biochemical availability of these elements, and the accessibility of calcium and phosphorus decreases followed by their reduction in blood, which results in such effect as hypocalcemia. However, the fact that the feeds contain sufficient amount of calcium, produces an illusion of calcium deficiency $[1,9]$.

It often happens that phosphorus deficiency is accompanied by the increase of inorganic phosphorus (inorg. P) in blood, which is explained at early stages by degradation of bone tissue osteoclasts, i.e. its mobilization from tail vertebra. In the autumn-winter period, sheep population of all natural and climate zones shows copper deficiency. This fact is consistent with the literature data provided by the researchers that the soil cover of Stavropol Krai lacks copper, and the food supply used in the sheep's diet is received locally $[1,9]$.

To prevent the deficiency of minerals, the complete study of regions, where sheep population is managed (quantitative assessment of elements in the soil cover, water and feeds), is necessary. The estimation of sheep population supply with various nutrients is only possible based on the detected interdependent factors connected with dietary and metabolism level.

Chemical elements included in the living organism structure are not synthesized in it but must be originated from feeds and other nutrients. There is a concept of essential elements of the animal body, i.e. the micronutrients which are deeply involved in the biochemical processes of the body. Changes in essential micronutrients supply shall not be studied in isolation from the general bio-geochemical situation. Both excess and deficiency may lead to metabolic disorders involving enzyme systems. Knowledge applied by veterinary specialists about the imbalance of various chemical elements in soil, water and feeds has formed the basis for raising the issue of endemic animal diseases diagnosis and prevention.

The feeds inadequacy often leads to undernutrition and the reduction of their value in the stall period, which causes the fatness reduction and therefore the decrease in mobility and activity, and the weakness of animals. General inspection and palpation of animals reveal ruffled coat, dry-looking and non-supple skin, and if the lack of nutrients becomes more intense, anemic mucous membranes (in more complicated forms even cyanosis and 
icterus) are observed.

The most efficient way to prevent the nutrients deficiency in all types of animals is placement in the leansoil of appropriate mineral fertilizers with different forms of essential nutrients. At the present day there is a shortage of such fertilizers as their production is not taken to the assembly line.The most available preventive measure today is the provision of mineral blocks containing relevant micronutrients. In case of abundance of a specific micronutrient in the feeds or in a diet, feeding with chemical antagonist is used.

The researches pursued at the premises of Agricultural Production Cooperative "Rodina" of the Arzgirsky District of Stavropol Krai were aimed at the implementation of diagnostic and preventive measures to recognize the incidence of disease caused by the lack of essential nutrients originated from the feeds in the autumn-winter period.

The experiment involved 146 fine-wool ewes and 160 lambs. All the animals used in the experiment were checked in the pre-lambing period, on the 20th, 40th, 60th day after the lambing; the lambs were checked at the age of 30,60 and 120 days. General inspection showed satisfactory condition of the investigated animals. Gain and development degree of the animal's organism were checked by weighting method $[10,11]$.

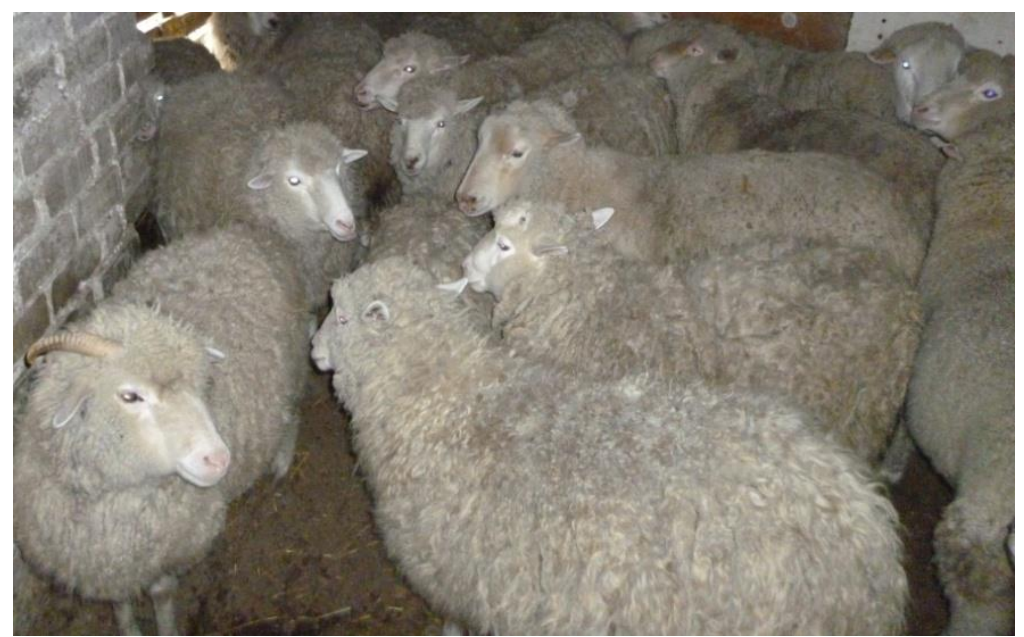

Fig. 1. Keeping sheep in the stall period.

Animals division into two groups was based on age, weight factor and other criteria.

The feeding of the experimental group consisted of the following diet: $1 \mathrm{~kg}$ of mixed herb and meadow hay of ordinary quality, 2-3 $\mathrm{kg}$ of wheat straw, $3 \mathrm{~kg}$ of corn silage and $300 \mathrm{~g}$ of concentrates; after the lambing sheep were additionally given $1 \mathrm{~kg}$ of hay, $1 \mathrm{~kg}$ of corn silage, $200 \mathrm{~g}$ of mixed feed and $200 \mathrm{~g}$ of linseed cake. The diet was balanced in accordance with feed units, protein, calcium, phosphorus and carotene.

The control group of sheep was fed in accordance with the diet approved in the farm which consisted of $2 \mathrm{~kg}$ of hay, $2 \mathrm{~kg}$ of straw, and $3 \mathrm{~kg}$ of corn silage.After the lambing, 1 $\mathrm{kg}$ of silage and $500 \mathrm{~g}$ of concentrated byproduct (irregularly)wereadded in the feeding diet. Salt blocks were given to sheep to appetite.

The analysis of data resulting from the study of the usage of the diet developed for the ewes from the experimental group revealed that the animals remained fleshed and active, and after the lambing an increased milking was evidenced while nursing the lambs. There were no evidenced changes in coat, mucous membranes, and any deviations of chemistry values on the side of vital body systems[11, 12].

Analysis of data received from the control group detected that in the second decade of the stall period the fatnessdecreased, the part of the group became inactive, which was 
evidenced by difficult raising, the animals often lay down to rest when walking, which made it impossible to tend them together with the experimental group. Future research detected observable decline in the sheep's health of the control group toward the end of the stall period. Their skin cover was dry to the touch and not supple enough, mucous membranes anemic, in some cases cyanosis and icterus were observed. Animals' body temperature lowered, pulse and heart tones were not apparent enough, nasal leakage in the form of malodorous mucus was observed. Moreover, there were cases of duodenum obstruction with wool in the lambs born from the sheep of the control group $[10,11,13]$.

Blood serum tests for calcium and phosphorus content in the formed sheep groups revealed their low concentration over the whole period of the experiment. The phosphorus level in blood serum proved to fall more significantly than in the control group in spring (late March and April) and reached the average index of $3.3 \mathrm{mg} \%$ (Table 1). The fixedfluctuations of inorganic phosphorus in blood serum resulted in significant changes in calcium and phosphorus proportion.

Table 1. Calcium and phosphorus content in sheep's blood serum (mol/L).

\begin{tabular}{ccccccccc}
\hline & \multicolumn{1}{c}{ Before lambing } & \multicolumn{7}{c}{ After lambing (days) } \\
\cline { 2 - 9 } Groups & $\mathrm{Ca}$ & $\mathrm{P}$ & $\mathrm{Ca}$ & $\mathrm{P}$ & $\mathrm{Ca}$ & $\mathrm{P}$ & $\mathrm{Ca}$ & $\mathrm{P}$ \\
\hline $\begin{array}{c}\text { Experi } \\
\text { ment }\end{array}$ & 12.3 & 7.8 & 11.8 & 7.2 & 12.2 & 7.3 & 12.1 & 6.0 \\
Control & 12.3 & 7.6 & 11.2 & 6.5 & 11.3 & 6.1 & 10.7 & 4.0 \\
\hline
\end{tabular}

The control group formed by the beginning of the experiment showed 1.5 to 2.0 ratio of calcium to phosphorus, and 3.4 to 4.0 by the end of the experiment. During the experiment, some ewes did not lamb, i.e. there was no crop. Clinical examination of the control sheep population detected the decreased muscle tone and conduction of nerve impulse, the sheep were weak and inactive.

The ewes from the experiment population kept the lactation level typical for summer season during 100 - 120 days, while the lactation of the ewes from the control group stopped by the 45 th -90 th day. Tabular data show that calcium and phosphorus content during the sheep's lactation depended on the feeding balance. The examination of the experimental group detected the kept muscle tone and normal conduction of nerve impulse, the sheep were lively and active.

Calcium and phosphorus content in the lactation period in the formed groups differed (Table2).

Table 2. Calcium and phosphorus content in sheep's milk (mmol/L).

\begin{tabular}{|c|c|c|c|c|c|c|c|c|}
\hline \multirow{3}{*}{ Groups } & \multicolumn{8}{|c|}{ Days after lambing } \\
\hline & \multicolumn{2}{|c|}{15} & \multicolumn{2}{|c|}{45} & \multicolumn{2}{|c|}{90} & \multicolumn{2}{|c|}{120} \\
\hline & $\mathrm{Ca}$ & $\mathrm{P}$ & $\mathrm{Ca}$ & $\mathrm{P}$ & $\mathrm{Ca}$ & $\mathrm{P}$ & $\mathrm{Ca}$ & $\mathrm{P}$ \\
\hline Experiment & 23.8 & 11.8 & 23.7 & 10.1 & 23.8 & 11.1 & 24.1 & 11.5 \\
\hline Control & 21.5 & 8.9 & 20.9 & 7.5 & 20.9 & 9.8 & 21.4 & 10.6 \\
\hline
\end{tabular}

Biochemical studies of calcium and phosphorus content in the lambs' blood serum show the same dynamics asfor the ewes, but such indexes proved to be significantly higher for the adult animals. The parallel trials revealthe higher level of phosphorus content in the lambs than in the ewes, provided that the lambs of the control group show its sharp and consistent decrease (Table3). 
Table 3. Calcium and phosphorus content in lambs' blood serum (mmol/L).

\begin{tabular}{cccccccc}
\hline \multirow{2}{*}{ Groups } & \multicolumn{2}{c}{30} & & & 60 & \multicolumn{2}{c}{120} \\
\cline { 2 - 7 } & $\mathrm{Ca}$ & $\mathrm{P}$ & $\mathrm{Ca}$ & $\mathrm{P}$ & $\mathrm{Ca}$ & $\mathrm{P}$ \\
\hline Experiment & 13.4 & 8.5 & 12.5 & 7.4 & 12.4 & 7.6 \\
Control & 13.2 & 8.4 & 12.1 & 7.0 & 12.0 & 4.2 \\
\hline
\end{tabular}

Norm: calcium 10-12.5; phosphorus 4.5-7.5.

As the table shows, the deficient feeding of the ewes from the control group led to the negative changes in the lambs resulting in hypophosphorosis.

The lambs born in the control group had growth and development retardation, had lower body weight gain in comparison with the one in the experimental group, and were more liable to skin diseases. The control group animals' examination and observation acknowledged satisfactory condition and the absence of any visible changes of the skin cover, while the control group sheep showed ruffle coat in shoulders and abdomen area. It should be emphasized that the animals managed according to the recommended diet had satisfactory body weight gain, calcium and inorganic phosphorus content in the blood serum, and were less liable to skin and cover diseases in the autumn-winter period $[12,14]$.

\section{Discussion}

The analysis of the received statistical material let us to submit the approximate content and to calculate the proportion of components for creep feeding blocks: table salt, sodium phosphate, sublimed sulfur, zinc sulfate, and potassium iodide for molding in blocks which can be used for feeding. The blocks composition considers the actual minerals content in the feeding regarding the specific features of different agroclimatic areas. The use of salt blocks in many ways shows tangible results with respect to the prevention of skin diseases in sheep population, especially those of parasitic origin. Their systematic use increases appetite, feed intake, all types of productivity and resistance indexes. Mineral blocks are transportable, storable, easy to use, and their usage improves the economic effectiveness.

\section{Conclusions}

- The conducted research detected disturbances of mineral metabolism in the control group's ewes and lambs as the result of the diet recommended by the farm, with more obvious phosphorus deficiency.

- The corrected diet we recommend makes it possible to regulate the mineral metabolism of the control group, both in mature animals and young stock, with respect to macronutrients (calcium and phosphorus).

- The conducted adjustment of the feeds with enough content of macronutrients made it possible to prevent skin diseases.

\section{References}

1. F. Wang, Z. Wang, C. Kou, et al., PLoS ONE, 11, 1 (2016)

2. D. Pal D, N. Gowda, C. Prasad, et al., Indian Journal of Animal Sciences, 79, 6, 615619 (2009)

3. K. Deghnouche, M. Tlidjane, T. Meziane, Research for Rural Developmen, 25, 9 
(2013)

4. B. Bagamaev, N. Fedota, E. Gorchakov, et al., Research Journal of Pharmaceutical Biological and Chemical Sciences, 9, 9, 1550-1555 (2018)

5. T. Glyzina, E. Matugina, B. Bagamaev, et al., IOP Conference Series: Earth and Environmental Science, 33, 1,12-25 (2016)

6. M. Kohler, F. Leiber, H. Willems, et al., Journal of Animal Science, 91, 11, 5259-5268 (2013)

7. S. Mir, H. Ahmed, A. Ganai, Y. Beigh, Biological Rhythm Research (2019) DOI: 10.1080/09291016.2019.1566992

8. R. Da Cruz, F. Rocha, C. Sena, et al., Ciencias Agrarias, 38, 5, 3085-3093 (2017)

9. K. Deghnouche, M. Tlidjane, T. Meziane, Research for Rural Development, 25, 9 (2013)

10. Y. Mekuriaw, B. Asmare, Agriculture and Food Security, 7, 1 (2018)

11. A. Filho, G. Carvalho, A. Pires, et al., Pereira F Journal of Animal Sciences, 27, 2, 284-289 (2014)

12. P. Kumar, K. Pasupathy, K. Kumar, et al., Indian Journal of Animal Research, 52, 4, 564-568 (2018)

13. A. Bhat, A. Ishfaq, A. Ganai, et al., Indian Journal of Animal Research, 52, 7, B-3321, 1010-1013 (2018)

14. X. Xie, J.-K. Wang, L. Guan, J.-X. Liu, Animal Science Journal, 89, 1, 122-131(2018) 